Boise State University

ScholarWorks

Materials Science and Engineering Faculty

Publications and Presentations

Department of Materials Science and Engineering

$10-1-2016$

Sample Preparation Techniques for Grain Boundary Characterization of Annealed TRISOCoated Particles

\author{
M. L. Dunzik-Gougar \\ Idaho State University \\ I. J. van Rooyen \\ Idaho National Laboratory \\ C. M. Hill \\ Idaho State University \\ T. Trowbridge \\ Idaho National Laboratory \\ J. Madden \\ Idaho National Laboratory \\ See next page for additional authors
}


Authors

M. L. Dunzik-Gougar, I. J. van Rooyen, C. M. Hill, T. Trowbridge, J. Madden, and J. Burns 


\title{
Sample Preparation Techniques for Grain Boundary Characterization of Annealed TRISO-Coated Particles
}

\author{
M. L. Dunzik-Gougar, ${ }^{a *}$ I. J. van Rooyen, ${ }^{b}$ C. M. Hill,,${ }^{\mathrm{a} b} \mathrm{~T}$. Trowbridge, ${ }^{\mathrm{c}}$ J. Madden, ${ }^{\mathrm{c}}$ and J. Burns ${ }^{\mathrm{d}, \mathrm{e}}$ \\ ${ }^{a} I d a h o$ State University, Department of Nuclear Engineering and Health Physics, Idaho Falls, Idaho 83209-8150 \\ ${ }^{h}$ Idaho National Laboratory, Fuel Performance and Design Department, Idaho Falls, Idaho 83415-6188 \\ 'Idaho National Laboratory, Fuel Fabrication and Characterization Department, Idaho Falls, Idaho 83415-6188 \\ ${ }^{d}$ Center for Advanced Energy Studies, Idaho Falls, Idaho 83401 \\ ${ }^{e}$ Boise State University, Department of Materials Science and Engineering, Boise, Idaho 83725-2090
}

Received September 29, 2015

Accepted for Publication May 13, 2016

http://dx.doi.org/10.13182/NT15-129

\begin{abstract}
Crystallographic information about layers of silicon carbide ( $\mathrm{SiC}$ ) deposited by chemical vapor deposition is essential to understanding layer performance, especially when the the layers are in nonplanar geometries (e.g., spherical). Electron backscatter diffraction (EBSD) was used to analyze spherical SiC layers using a different sampling approach that applied focused ion beam (FIB) milling to avoid the negative impacts of traditional sample polishing and address the need for very small samples of irradiated materials for analysis. The mechanical and chemical grinding and polishing of sample surfaces can introduce lattice strain and result in the unequal removal of $\mathrm{SiC}$ and the surrounding layers of different materials due to the hardness differences among these materials. The nature of layer interfaces is thought to play a key role in the performance of $\mathrm{SiC}$; therefore, the analysis of representative samples at these interfacial areas is crucial. In the work reported herein, a FIB was employed in a novel manner to prepare a more representative sample for EBSD analysis from tristructural-isotropic layers that are free of effects introduced by mechanical and chemical preparation methods. In addition, the difficulty of handling neutron-irradiated microscopic samples (such as those analyzed in this work) has been simplified using pretilted mounting stages. The results showed that while the average grain sizes of samples may be similar, the grain boundary characteristics can differ significantly. Furthermore, low-angle grain boundaries comprised $25 \%$ of all boundaries in the FIB-prepared sample compared to only $1 \%$ to $2 \%$ in the polished sample from the same particle. This study demonstrated that the characterization results from FIB-prepared samples provide more repeatable results due to the elimination of the effects of sample preparation.
\end{abstract}

Keywords - Silicon carbide, focused ion beam, grain boundary character.

Note - Some figures may be in color only in the electronic version.

\section{INTRODUCTION}

A significant challenge for next-generation, hightemperature nuclear reactor designs is the availability of new materials compatible with extreme conditions. Developing this type of material is the focus of work presented here. Silicon carbide $(\mathrm{SiC})$ has extraordinary physiochemical

*E-mail: mldg@isu.edu properties, including chemical stability, thermal and radiation resistance, high resistance to oxidation, high thermal conductivity, and high mechanical strength. Dimensional stability under high temperature (i.e., maximum linear expansion of $0.7 \%$ at $250^{\circ} \mathrm{C}$ ) and irradiation conditions further make $\mathrm{SiC}$ a material of interest. Of greatest significance for the nuclear industry, SiC serves as the main barrier to fission product release in the high-temperature gas reactor design. Fission product migration, particularly 
silver $(\mathrm{Ag})$ migration, through the $\mathrm{SiC}$ layer in tristructuralisotropic (TRISO)-coated fuel (see Fig. 1) has been internationally investigated for more than 40 years (Refs. 1 and 2); however, past modeling efforts have failed to fully describe the measured $\mathrm{Ag}$ release. ${ }^{3}$ One of several migration mechanisms studied is migration via $\mathrm{SiC}$ grain boundaries. A greater understanding of the nature of $\mathrm{SiC}$ grain boundaries is essential to understanding and preventing fission product migration in this mode.

Electron backscatter diffraction (EBSD) is a practical characterization technique for obtaining crystallographic information, including crystal type, orientation, grain boundary characteristics, grain size distribution, and texture. These measurements are obtained from small areas using scanning electron microscopy (SEM). The ability of this technique to perform scanning over a wide range of magnifications makes it possible to investigate the microstructure down to nano-levels when needed. ${ }^{4}$ Data from this type of analysis provide details about the distribution of high-angle grain boundaries, which can be used to test the hypothesis of $\mathrm{Ag}$ transport along grain boundaries. ${ }^{4-7}$

Because $\mathrm{Si}$ and $\mathrm{C}$ are fairly light elements, the signal generated for EBSD collection is weak. However, the unirradiated $\mathrm{SiC}$ materials examined to date possessed fairly well-preserved microstructures and were measureable. However, because a crystal structure is stressed mechanically by heat treatment or by neutron exposure, the Kukuchi patterns (i.e., lines) will become weak. As the material approaches an amorphous state, the lines may not exist at all. This behavior provides an additional challenge for the collection of EBSD data on irradiated SiC layers. Reliable EBSD analysis of a polycrystalline material

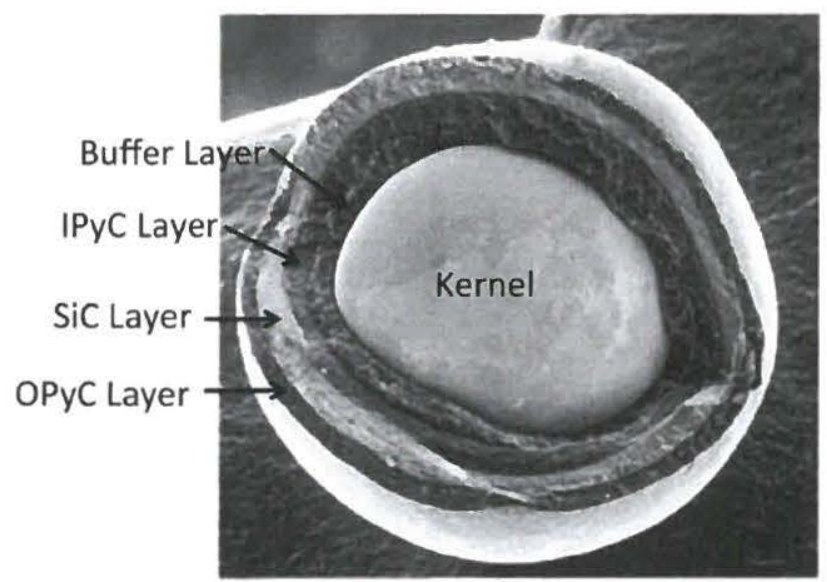

Fig. 1. SEM image of a TRISO-coated particle showing the kernel at center surrounded by three types of layers: a porous carbon buffer surrounding the kernel and pyrolytic carbon layers (IPyC and $\mathrm{OPyC}$ ) sandwich layers around the $\mathrm{SiC}$ layer. (such as $\mathrm{SiC}$ ), whether it is irradiated or not, requires a smooth surface. ${ }^{7}$ Typically, the methods of sample preparation for the analysis of an $\mathrm{SiC}$ layer consist of mechanically grinding a sample TRISO-coated particle that is embedded in epoxy resin down to a hemispherical cross section. Subsequent fine polishing can be accomplished using various methods, ${ }^{7-10}$ including that reported by Tan et al., ${ }^{10}$ who sequentially used diamond paste, alpha alumina, and colloidal silica solutions with good results. Nevertheless, in many instances, researchers studying TRISO particles have encountered difficulties when mechanically and chemically grinding and polishing sample surfaces because these methods can introduce lattice strains and result in the unequal removal of the $\mathrm{SiC}$ layer and surrounding pyrocarbon layers. This phenomenon is illustrated in the micrograph of a traditionally polished TRISO particle in Fig. 2.

Focused ion beam (FIB) milling can sputter materials of differing hardness values in a highly localized manner using a beam probe with a diameter of approximately $5 \mathrm{~nm}$. Consequently, FIB cross sectioning through materials of differing hardness has a much better outcome compared to using mechanical methods." Kirchhofer et al. ${ }^{8}$ used a FIB to raster across the surface of a section of a mounted, coated TRISO particle that previously had been mechanically polished in preparation for analysis. The FIB removed the rounded edges between the different layers, creating a smooth surface suitable for EBSD analysis. However, it is unclear if the depth of material removal by FIB results in the removal of all material mechanically affected by the polishing/grinding process.

To address the problems caused by mechanical polishing, van Rooyen et al. ${ }^{12}$ used a FIB to mill into the particle and extract a sample from within the TRISO-coated layers, well beneath (at least $35 \mu \mathrm{m}$ from the cross-sectioned surface) any damage from material grinding. This difference in the application of the FIB and relative EBSD



Fig. 2. SEM image $(3300 \times)$ of the sample Eref SiC layer with rounded edges at the IPyC and OPyC layer boundaries after traditional polishing (adapted from Ref. 9). 
sample location is visualized in Fig. 3. Additionally, this sampling technique facilitates the EBSD analysis of neutron-irradiated $\mathrm{SiC}$ layers, for which very small samples are required. It is necessary to prepare ever-smaller irradiated samples to accommodate activity and dose limits in characterization facilities.

In general, handling very small samples for analysis is difficult. Mounting and proper alignment are difficult without the aid of a high-powered microscope. For example, Helary et al. ${ }^{13}$ reported an awkward ex situ transfer of a cross-sectioned particle from epoxy to the SEM sample stage; it was necessary to align the cross-sectioned face to be exactly parallel to the sample stage. The FIB removal of a small section of the TRISO layers by van Rooyen et al. $^{12}$ addressed the need for very small samples of irradiated material, which are much more easily aligned than whole particles. In addition, the small sample allows for the successive FIB removal of layers to create threedimensional images. Such successive milling and imaging is a very cumbersome process when using whole particles.

The primary objective of the work presented in this paper is to demonstrate the specific FIB technique for the removal of a small but representative sample for an outof-pile study of separate effects. The EBSD results of samples prepared by both traditional polishing and by the
FIB milling and polishing technique are compared. The general use of FIB lift-out techniques to prepare EBSD samples is well-established ${ }^{14-18}$; however, the authors have demonstrated the use of FIB to access and extract a representative sample from within a material that was exposed for analysis by mechanical and chemical polishing. While mechanical and chemical polishing are necessary steps, the result is a material unsuitable for EBSD analysis due to rounded edges between layers of differing hardness and due to damage of the crystal structure. When FIB milling is used well below the surface of the material to be analyzed, a sample free of the effects of chemical and mechanical polishing is produced for EBSD analysis. Such samples will also be amenable to additional analyses, such as the three-dimensional rendering of grain boundary characteristics via successive FIB milling and EBSD characterization.

\section{MATERIALS AND METHODS}

\section{II.A. Sample Description}

The coated particles used to develop the modified FIB sample preparation method had nonfuel kernels (composed of $\mathrm{ZrO}_{2}$ ) surrounded consecutively by layers of

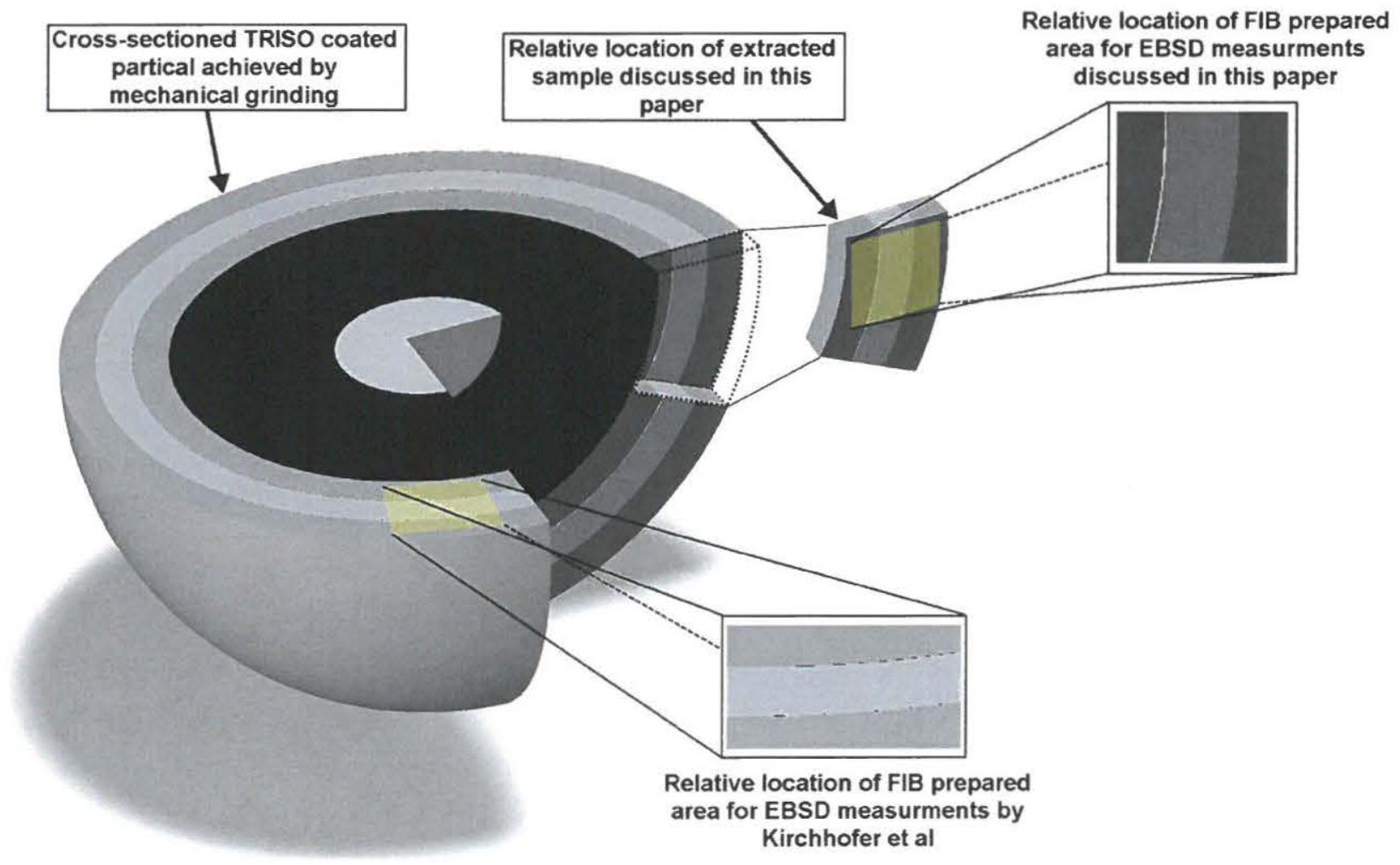

Fig. 3. Illustration showing a cross-sectioned, TRISO-coated fuel particle and the relative locations of samples removed via FIB for EBSD analysis by Kirchhofer et al. ${ }^{8}$ and van Rooyen et al. ${ }^{12}$ 
porous carbon buffer, inner pyrolytic carbon (IPyC), $\mathrm{SiC}$, and outer pyrolytic carbon $(\mathrm{OPyC})$. The $\mathrm{SiC}$ layer was applied via chemical vapor deposition at $1510^{\circ} \mathrm{C}$ at a deposition rate of $0.24 \mu \mathrm{m} / \mathrm{min}$ at the Advanced Coating Facility of the South African Nuclear Energy Corporation. The coated particles were suspended in an epoxy resin and mechanically thinned to hemispheres using a Buehler-Beta grinder polisher, thus exposing the various coating layers. Finally, the particles were polished using a $0.05-\mu \mathrm{m}$ colloidal silica suspension. ${ }^{9}$ The samples were further mounted in an epoxy resin for transport and analyses.

\section{II.B. Sample Preparation Technique}

The sample extraction method begins by placing a mounted cross-sectioned hemispherical fuel particle into the FIB/SEM system. The surrogate fuel samples used in this project were prepared and analyzed using an FEI Quanta 3D SEM-FIB instrument for the high-resolution imaging and milling of specimens. This instrument was also equipped with platinum and carbon gas-injection systems for deposition specimen marking and probe welding as well as an OMNI Probe for the in situ lift-out of samples from a given specimen.

The gas-injection system is used to deposit a platinum marker on a random portion of the exposed $\mathrm{SiC}$ ring. This marker is used as a guide to mill the surrounding material to expose a surface segment of the $\mathrm{SiC}$ layer between the IPyC and the OPyC layers that was not exposed to grinding damage during the preparation of the original cross section. The segment of interest for EBSD analysis is the side face of the wedge formed by cutting into the surface on either side of the platinum rectangle (see Fig. 4).

To reach the desired $35-\mu \mathrm{m}$ depth from the top face of the cross-sectioned particle, wedge-shaped trenches are excavated from the material starting just above and below the platinum marker while the stage is tilted at $52 \mathrm{deg}$. At this angle (i.e., $52 \mathrm{deg}$ from the electron beam), the ion beam is normal to the sample surface, with beam coincidence at the sample eucentric height of about $10 \mathrm{~mm}$. This tilt makes room for the FIB to cut the bottom sample surface on both sides at a 52-deg angle while the sample is in an upright position. The volume to be milled is programmed into the system as a box. If a depth of $35 \mu \mathrm{m}$ is desired, the upper box surface rectangle would need to be drawn with sufficient dimensions to compensate for the redeposition of milled material that is not carried away in the vacuum system. When the dimensions of the upper rectangular layer of the trench are established using patterning tools, the ion beam is ready to raster across the chosen rectangle to sputter material as it progresses.

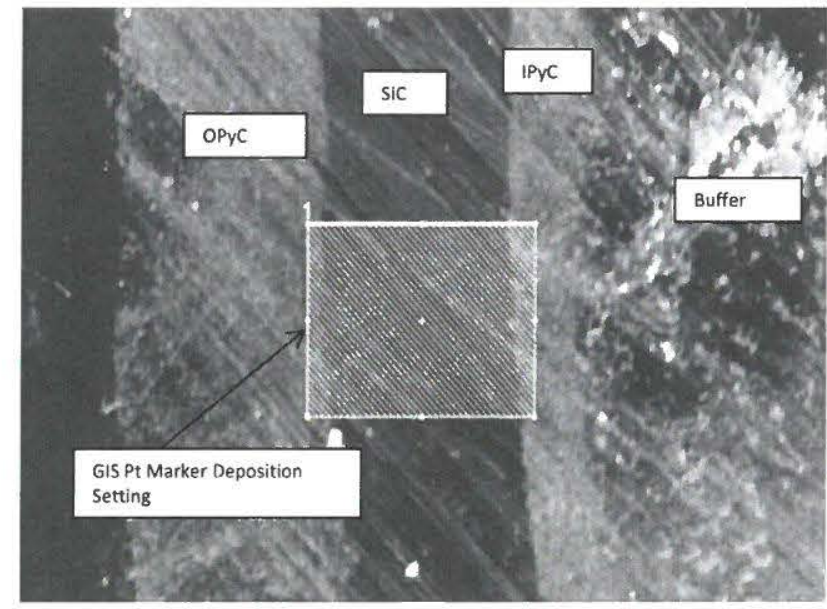

(a)

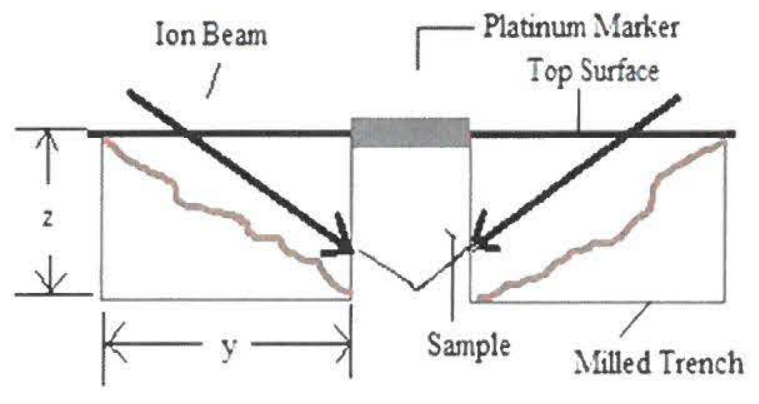

Side View

(b)

Fig. 4. (a) SEM image of the dimension settings for a gas injection system-deposited platinum marker used in preparation for ion beam milling segment extraction of the sample SiC layer. (b) Schematic of the FIB sample extraction step. The electron beam, not shown in the image, is normally oriented with respect to the sample surface.

Debris buildup is a common occurrence in deep trenches and is sometimes termed redeposition curtain. The $x$-dimension of the trench is set by visual inspection. The $y$-dimension in this instance was given a dimension value of one and a half times the desired depth. Figure 5 illustrates the beginning phase of milling and the completed trenches for the extraction of a sample of $\mathrm{SiC}$ layer from a TRISO particle.

Note the difference in milling morphology between the surrounding pyrocarbon layers and the $\mathrm{SiC}$ layer in Fig. 5a. The SiC layer grains appear to have more spherical shapes compared to the long columnar grains along the radial direction, which are typical of the chemical vapor deposition-coating process. In addition, the $\mathrm{SiC}$ grains do not appear to increase in size from the IPyC layer to the OPyC layer. 


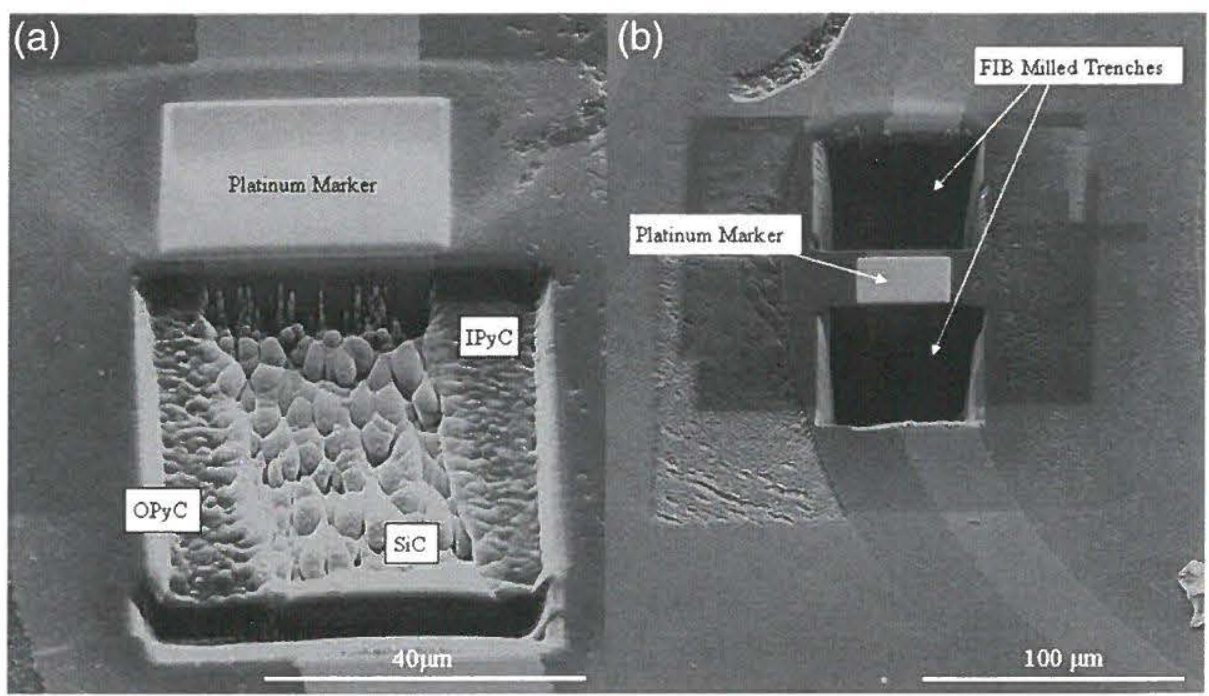

Fig. 5. SEM images (a) at the beginning stage of ion beam bulk milling for sample extraction of an SiC layer from a TRISO-coated particle and (b) at the completion of bulk-milled trenches above and below the platinum marker.

Figure 6a shows the resulting debris formation in the bulk-milled trenches and the redeposition of material onto the surface of interest. The specimen platform is tilted to 52 deg to achieve a 90-deg angle between the ion beam and specimen surface during bulk milling. To clean the debris off the face of interest, the platform is tilted an additional $2 \mathrm{deg}$ to $54 \mathrm{deg}$. A small rectangle is drawn near the edge of the platinum marker with a patterning tool, and the ion beam is employed to sputter material to the required depth within the chosen area. This procedure exposes the $\mathrm{SiC}$ layer (see Fig. 6b).

As shown in Fig. 6, a small arm of material is left on one side to stabilize the specimen during the cutting of the bottom portion for final sample separation.

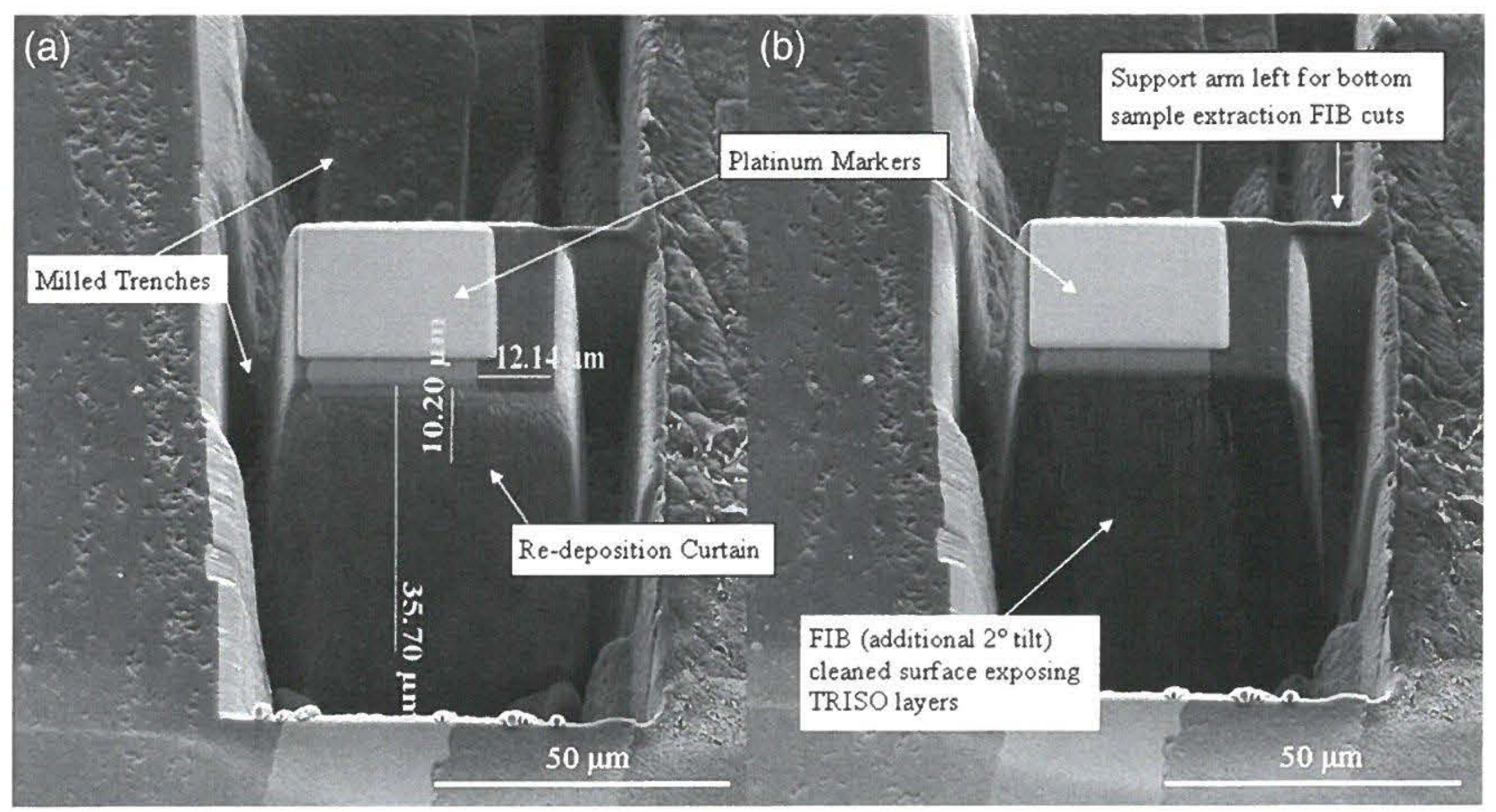

Fig. 6. SEM images of a TRISO-coated particle SiC layer sample (a) after the formation of bulk-milled trenches and side cuts and (b) after the ion beam milling of a debris curtain from the surface of interest at a specimen tilt angle of 54 deg from vertical. 
For cutting, the stage is tilted back to the upright position to allow the ion beam, which is at an angle of $52 \mathrm{deg}$ with respect to vertical, to slice segments at the bottom of the sample in preparation for removal. Figure 4 shows a sketch illustrating this step. The ion beam consecutively passes unhindered through each milled, wedge-shaped trench to form a V-shaped cut into the bottom of the sample when viewed from the side. The sample is rotated $180 \mathrm{deg}$ between the two ion beam cuts. The arm on one side continues to hold the sample in place while an OMNI probe needle used for lift-out is welded to the sample using the platinum deposition feature.

EBSD analysis requires that the normal surface of evaluation be tilted $70 \mathrm{deg}$ with respect to vertical. The stage control will not tilt the full $70 \mathrm{deg}$. Attaching the sample to a grid clamped into place by a 45 -deg pretilted mount accommodates this requirement. The next step welds the extracted sample onto a copper half grid that contains a series of labeled posts for attaching FIB liftouts. The stage is then tilted $7 \mathrm{deg}$. This adjustment plus the pretilt of 45 deg puts the sample in position for FIB polishing of the sample surface (i.e., close to parallel with the ion beam). A series of gradually decreasing currents $(30,15,7,1,0.3$, and $0.1 \mathrm{nA}$ and $48 \mathrm{pA}$ at $5 \mathrm{keV})$ was used to achieve a gradual reduction in hills and valleys on the sample surface for a smooth finish. The sample surfaces before and after FIB polishing are shown in Fig. 7. Sample extraction, polishing, and EBSD scanning were performed in the same chamber.

\section{II.C. EBSD Data Collection Parameters}

For all samples prepared via traditional polishing, grains at the IPyC-SiC and OPyC-SiC boundaries were excluded from the EBSD analyses due to lack of clarity from rounded edges.
A fourth data set was obtained from a sample prepared with the FIB method described above. Boundary grains were included in the analysis.

The traditionally polished samples were analyzed using an FEI Quanta 650 FEG FIB-SEM, and the FIBpolished samples were analyzed with an FEI Quanta 3D FEG FIB-SEM. Both instruments were equipped with TSL Hikari EBSD systems. All EBSD scans were performed using a hexagonal grid pattern to maximize the number of data points collected within the scanned area. The area for EBSD analysis was selected with an initial step size of $50 \mathrm{~nm}$ for the first measurement and then standardized at $0.1 \mu \mathrm{m}$ for the mapping chosen for all subsequent analyses. The scanned areas were approximately $13 \mu \mathrm{m} \times 36 \mu \mathrm{m}$ for the traditionally polished samples and $45.70 \mu \mathrm{m} \times 31.70 \mu \mathrm{m}$ for the FIB-polished samples. The accelerating voltage was $20 \mathrm{kV}$. All data were cleaned via grain confidence index standardization and neighbor orientation.

\section{RESULTS AND DISCUSSION}

The EBSD results for the SiC layer samples of the same particle batch (Eref) are presented. Three sets of data were obtained from samples prepared via traditional polishing. Two of these three data sets were obtained from different locations in the same particle (Eref1.1 and Eref1.2) and were compared to results from a second particle (Eref2) to investigate the extent that measurements at one location in one particle of a batch were representative of batch properties.

Figures 8 and 9 show the grain size, character, and orientation data for the $\mathrm{SiC}$ layer of TRISO-coated particles from the same batch (Eref) prepared by traditional polishing (Eref1.1, Eref1.2, and Eref2) and FIB (Eref3FIB).

While three sets of polished sample diameter data did not allow for a conclusive determination, these data clearly do not rule out the possibility that limited particle



Fig. 7. SEM images of the sample surface (a) before FIB polishing and (b) after FIB polishing. 


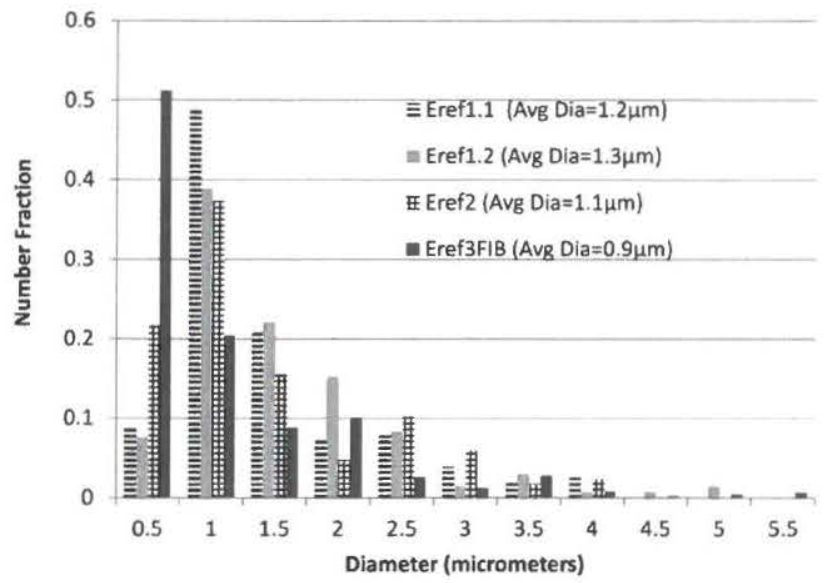

Fig. 8. SiC layer grain size (i.e., diameter) distribution via EBSD analysis of coated particles Erefl (locations 1 and 2) and Eref2, prepared with traditional polishing, and Eref3FIB, prepared via FIB.

measurements can represent the properties of a batch. The average $\mathrm{SiC}$ grain diameter according to these data was $1.2 \mu \mathrm{m}$ with a spread of only $\pm 0.1 \mu \mathrm{m}$. In contrast, the average diameter determined via analysis of the FIBprepared sample was $0.90 \mu \mathrm{m}$, which was lower than the three-measurement average by $25 \%$. The reason for the lower average diameter may be the inclusion of edge (i.e., boundary) grain sizes. These grains appear to be smaller than those toward the center of the $\mathrm{SiC}$ layer (see Fig. 9d).

In the EBSD images of traditionally polished samples (Figs. 9a, 9b, and 9c), the pyrocarbon layers would appear at the top and bottom of the images; however, because edge grains of the $\mathrm{SiC}$ layer were excluded from the analysis, the dark areas at the top and bottom are merely an artifact of the analysis rather than an indication of the start of a pyrocarbon layer. In contrast, the transition between the $\mathrm{SiC}$ and pyrocarbon layer (from right to left) and the $\mathrm{SiC}$ layer edge grains are clear in the FIB sample (see Fig. 9d). While the orientations of the polished and FIB samples are different, it should be noted that the SiC layers are theoretically isotropic.

Grain boundary character distributions for the samples are presented in Figs. 10 and 11. A statistically significant difference is noted between the $\mathrm{SiC}$ grain characteristics determined via analysis of polished samples (Fig. 10) and those determined from the FIB-prepared sample (Fig. 11). The most significant difference is the relative quantity of low-angle grain boundaries, which comprise $25 \%$ in the FIB-prepared sample (Fig. 11) and only $1 \%$ to $2 \%$ in the polished sample (Fig. 10). This difference is almost certainly due to the exclusion of layer edge grains in the polished sample analysis. The $\mathrm{SiC}$ grains at the pyrocarbon boundaries are notably smaller
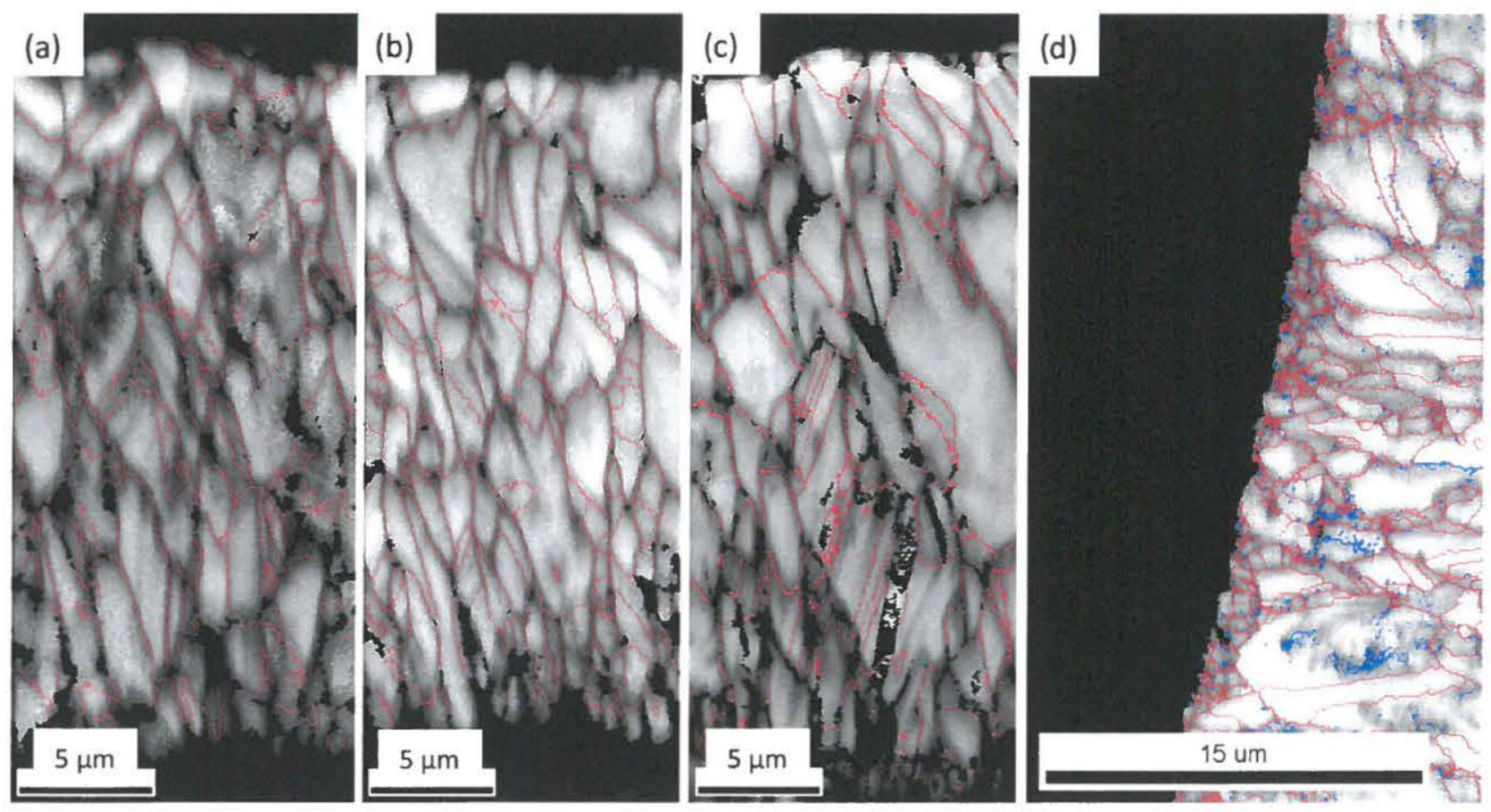

Fig. 9. EBSD images of SiC layer grains in samples (a) Eref1, location 1; (b) Eref1, location 2; and (c) Eref2, prepared with traditional polishing; and (d) Eref3FIB, prepared via FIB. 


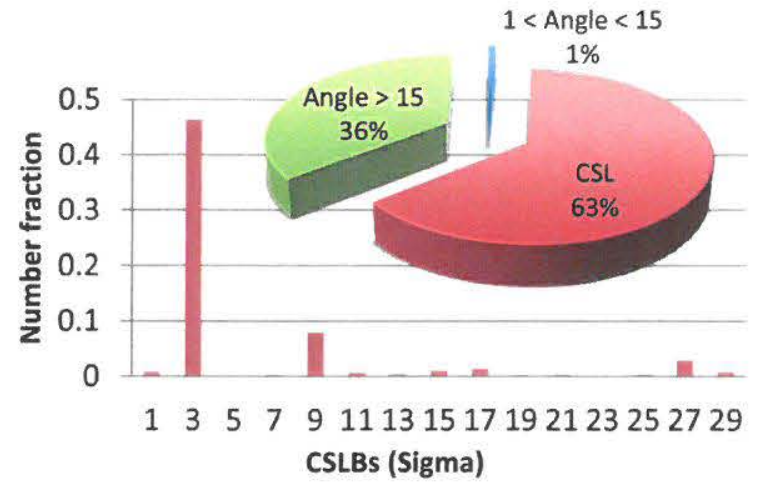

(a)

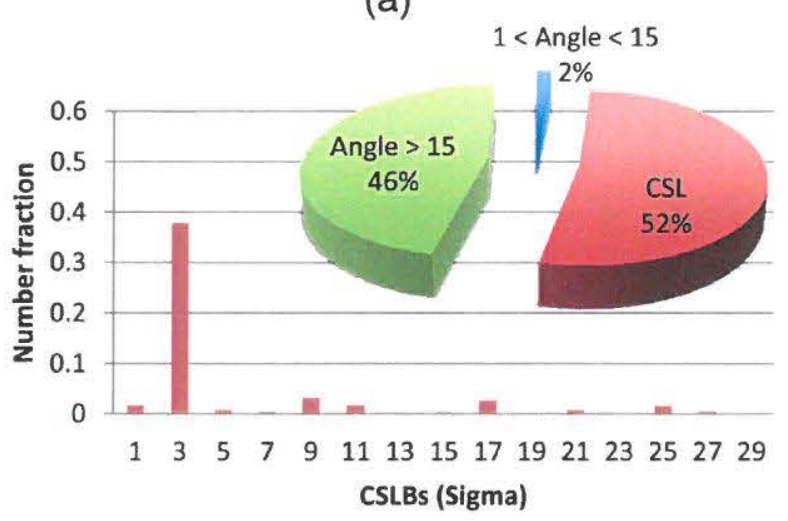

(b)

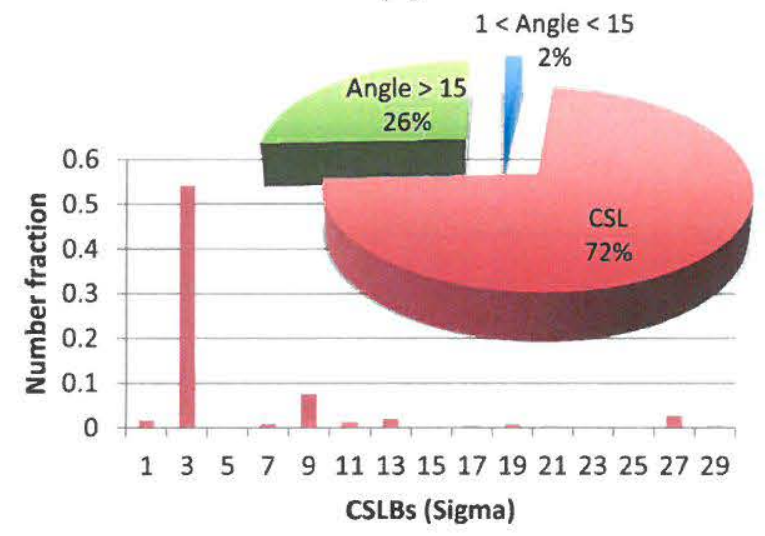

(c)

Fig. 10. SiC layer grain boundary type distribution determined from EBSD analysis of (a) coated particle Eref1, location 1, prepared with traditional polishing; (b) coated particle Eref1, location 2, prepared with traditional polishing; and (c) coated particle Eref2, prepared with traditional polishing.

than those at the layer interior, which is clearly visible in Fig. 9d.

For all samples, the percentage of coincidence site lattice (CSL) grain boundaries was the single largest category. For the polished samples, the CSL category was a

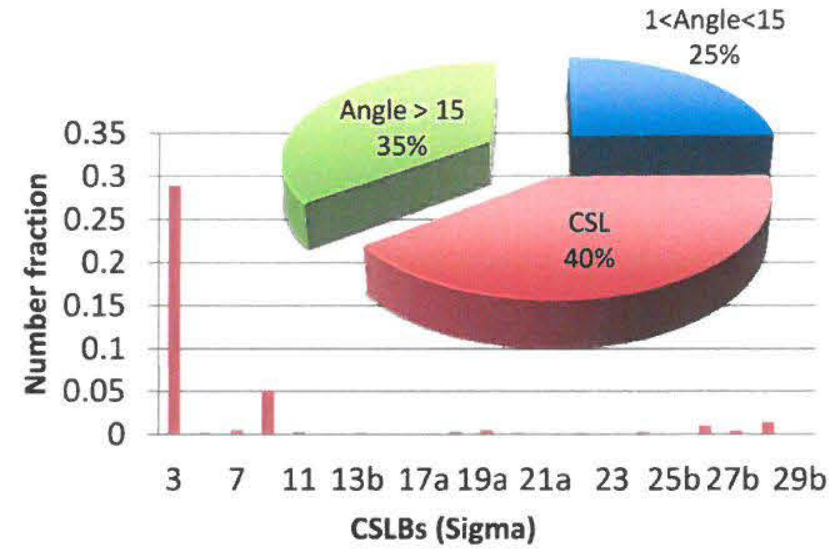

Fig. 11. SiC layer grain boundary type distribution determined from EBSD analysis of the coated particle Eref3FIB, prepared via the FIB method.

majority (i.e., ranging from $52 \%$ to $72 \%$ ); however, it was only $40 \%$ for the FIB-prepared sample. The exclusion of layer edge grains from the polished sample analysis may have biased the grain boundary character data toward a relatively larger average percentage of CSL boundaries. The spread of $52 \%$ to $72 \%$ of CSL grain boundaries in the polished samples may be attributed to an insufficient or nonhomogeneous polishing technique and does not necessarily represent a variation in the parameters of the batch of coated particles. Future analysis and interpretation will include binning the data as a function of distance from the interface.

Figure 12 shows the pole figures for each $\mathrm{SiC}$ analysis data set. In Fig. 12, the scale represents multiples of a random distribution in that sample. While the results indicate that none of the samples are particularly textured, there is a notable difference between the relative intensities of the grain orientations for the polished samples (maximum $=11$ to 13 ) and those for the FIB-prepared sample (maximum $=4$ ). This implies that a larger percentage of grains in the polished samples have a particular orientation, and that the FIBprepared sample is less textured than the polished samples. Again, this difference may be due to including layer edge grains in the FIB sample. The edge grains in Fig. $9 \mathrm{~d}$ are not only smaller than grains deeper in the $\mathrm{SiC}$ layer, but also less consistently oriented. A dominant $\mathrm{SiC}$ grain orientation is undesirable because it would facilitate the migration of fission product species along grain boundaries.

\section{SUMMARY AND CONCLUSIONS}

A method for the representative sample milling and polishing of damaged and dissimilar layers using FIB 

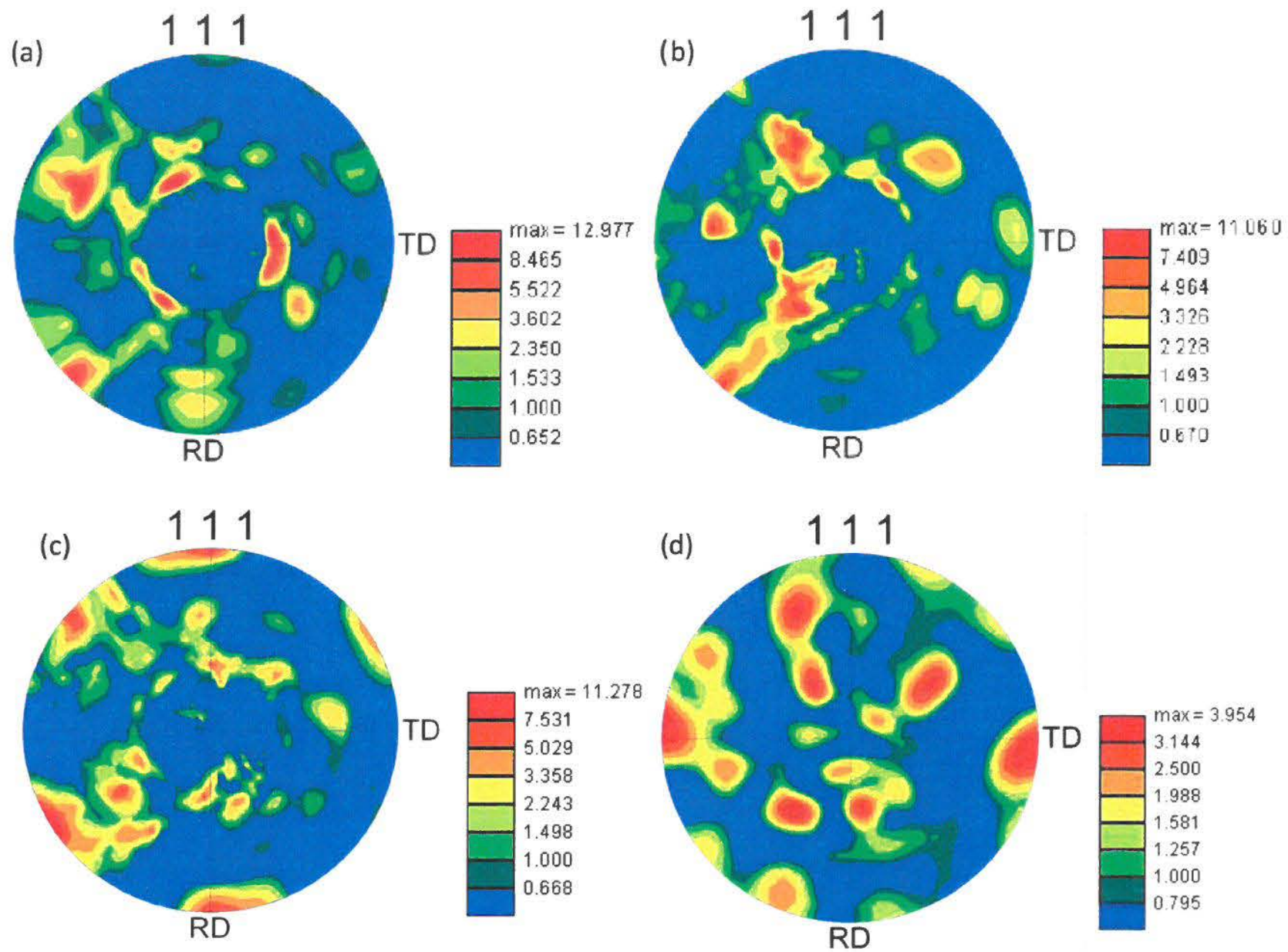

Fig. 12. SiC layer crystal orientation in the 111 direction determined via EBSD analysis of coated particle (a) Erefl, location 1; (b) Eref1, location 2; and (c) Eref2, prepared with traditional polishing; and (d) Eref3FIB, prepared via the FIB method. ( $\mathrm{TD}=$ tangent to the surface of the particle in the plane of the cross section and $\mathrm{RD}=$ normal to the plane of the cross section.)

extraction has been demonstrated and shows promise for materials containing components with different hardness values and for future application to neutron-damaged materials. The rounding and damage at the material edge that occurs from traditional sample polishing can be avoided, resulting in more complete and accurate sampling.

In addition to demonstrating the effects of different sample preparation techniques on the quality of the characterization results, comparative grain character analyses of TRISO particles from the same production batch were reported. Three of the data sets were obtained from traditionally polished $\mathrm{SiC}$ layers with rounded edges due to the polishing process. As such, the edge grains were not included in the final data analysis. In contrast, the FIB-milled, polished, and extracted sample had pristine edges, and all edge grains were included. In addition, the small size of the extracted sample allows the analyses of neutron-irradiated materials, which is the fate of the TRISO particles in this study. Small samples can be difficult to align properly for EBSD analysis; therefore, pretilted mounts allow for reliable and easier alignment of the $\mathrm{SiC}$ layer samples, resulting in viable analyses.

While more data are required to make a conclusive statement about the cause of differences in the resulting data, it would seem that including the edge grains had a significant impact on the average grain characteristics.

This study showed that the average grain sizes of samples can be similar even when the grain boundary characteristics differ significantly. Furthermore, it was found that low-angle grain boundaries comprise $25 \%$ in the FIB-prepared sample versus only $1 \%$ to $2 \%$ in the polished sample from the same particle. The findings indicated that characterizing FIB-prepared samples provides more repeatable results because the effects of sample preparation are eliminated. 


\section{Acknowledgments}

This work was sponsored by the U.S. Department of Energy, Office of Nuclear Energy, under U.S. Department of Energy Idaho Operations Office Contract DE-AC07-05ID14517, as part of the Very High Temperature Reactor Development Program and as part of an Advanced Test Reactor Nuclear Science User Facilities Experiment. We thank B. Coryell for his work on Fig. 3 and J. I. Cole for his review of this paper.

\section{References}

1. I. J. VAN ROOYEN et al., "Progress in Solving the Elusive $\mathrm{Ag}$ Transport Mechanisms in TRISO-Coated Particles: What is New?" Proc. 7th Int. Topl. Mtg. High Temperature Reactor Technology (HTR 2014), Weihai, China, October 27-31, 2014, paper HTR2014-31261.

2. W. AMIAN and D. STÖVER, "Diffusion of Silver and Cesium in Silicon-Carbide Coatings of Fuel Particles for High-Temperature Gas-Cooled Reactors," Nucl. Technol., 61, 475 (1983); http://dx.doi.org/10.13182/NT61-475.

3. B. P. COLLIN et al., "Comparison of Fission Product Release Predictions Using PARFUME with Results from the AGR-1 Irradiation Experiment," Proc. 7th Int. Topl. Mtg. High Temperature Reactor Technology (HTR 2014), Weihai, China, October 27-31, 2014.

4. A. WILKINSON, J. BRITTON, and T. BEN, "Strains, Planes, and EBSD in Materials Science," Mater. Today, 15, 9, 366 (2012); http://dx.doi.org/10,1016/S1369-702l(12) 70163-3.

5. E. LOPEZ-HONORATO et al., "Silver Diffusion in Coated Fuel Particles," J. Am. Ceram. Soc., 93, 10, 3076 (2010); http://dx.doi.org/10.1111/j.1551-2916.2010.04055.x.

6. I. J. VAN ROOYEN, Y. Q. WU, and T. M. LILLO, "Identification of Silver and Palladium in Irradiated TRISOCoated Particles of the AGR1 Experiment," J. Nucl. Mater., 446, 178 (2014); http://dx.doi.org/10.1016/j.jnucmat.2013. 11.028 .

7. S. SHIH, M. PARK, and D. J. H. COCKAYNE, "The Interpretation of Indexing of High $\Sigma$ CSL Boundaries from Ceramics," J. Microsc., 227, 309 (2007); http://dx.doi.org/ 10.1111/j.1365-2818.2007.01815.x.

8. R. KIRCHHOFER et al., "Microstructure of TRISO-Coated Particles from the AGR-1 Experiment: SiC Grain Size and Grain Boundary Character," J. Nucl. Mater., 432, 127 (2013); http://dx doi org/10.1016/j.jnucmat.2012.08.052.
9. I. J. VAN ROOYEN et al., "Effects of Phosphorous-Doping and High Temperature Annealing on CVD Grown 3C-SiC," Nucl. Eng. Des., 251, 191 (2012); http://dx.doi.org/10.1016/ j.nucengdes.2011.09.066.

10. L. TAN et al., "EBSD for Microstructure and Property Characterization of the SiC-Coating in TRISO Fuel Particles," J. Nucl. Mater., 372, 400 (2008); http://dx.doi.org/ 10.1016/j.jnucmat.2007.04.048.

11. Y. CHEN and X. ZHANG, "Focused Ion Beam Technology and Application in Failure Analysis," Proc. 11th Int. Conf. Electronic Packaging Technology \& High Density Packaging, Xi-an, China, August 16-19, 2010, paper 110.

12. I. J. VAN ROOYEN et al., "On Techniques to Characterize and Correlate Grain Size, Grain Boundary Orientation and the Strength of the SiC Layer of TRISO-Coated Particles: A Preliminary Study," Proc. 6th Int. Topl. Mtg. High Temperature Reactor Technology HTR2012, Tokyo, Japan, October 28-November 1, 2012, paper HTR-3-024.

13. D. HELARY et al., in Proc. Second Int. Topl. Mtg. High Temperature Reactor Technology, Beijing, China, September 22-24, 2004, paper B07.

14. J. R. MICHAEL and S. V. PRASAD, "Advances in Instrumentation and Techniques-Focused Ion Beam (FIB)/Dual Beam Applications and Techniques in Biological and Physical Sciences, FIB Preparation of Samples for EBSD: Applications to Wear Studies of MEMS," Microsc. Microanal., 10, Suppl. S02, 1130 (2004); http://dx doi.org/10. $1017 /$ S1431927604882631.

15. W. XU et al., "Techniques for Generating 3-D EBSD Microstructures by FIB Tomography," Mater. Charact., 58, 10, 961 (2007); http://dx.doi.org/10.1016/j.matchar.2006. 10.001 .

16. A. J. SCHWARTZ et al., Electron Backscatter Diffraction in Materials Science, Technology and Engineering, Springer Science and Business Media (Mar. 2010).

17. B. MATTHEY and S. HOHN, "High-Resolution Analysis of Interfaces by FIB/STEM and EBSD," in "IKTS Annual Report 2013/2014," p. 104, Fraunhofer IKTS; http://www.ikts. fraunhofer.de/content/dam/ikts/kommunikation/publikationen/ annual_reports/jb2013/Fraunhofer_IKTS_2013_Annual_Report. pdf (current as of Sept. 29, 2015).

18. P. WOO, "Applications of EBSD Using both Broad and Focused Ion Beam Milling as Sample Preparation Technique," Proc. SMCr. Séptimo Congreso Nacional de Cristalografiia, Villahermosa, Mexico, May 4-9, 2014. 